Open Access

\title{
Cryopreservation of equine mesenchymal stem cells in $95 \%$ autologous serum and $5 \%$ DMSO does not alter post-thaw growth or morphology in vitro compared to fetal bovine serum or allogeneic serum at 20 or $95 \%$ and DMSO at 10 or $5 \%$
}

Alexis Mitchell', Kristen A. Rivas ${ }^{1}$, Roger Smith $1 \|^{2}$ and Ashlee E. Watts ${ }^{1 *}$

\begin{abstract}
Introduction: Equine superficial digital flexor tendon injury is a well-accepted model of human tendon injury and is routinely treated with local injections of autologous mesenchymal stem cells (MSCs). Identification of a clinically safe medium for short-term cryopreservation of MSCs prior to cell implantation would streamline laboratory and clinical procedures for autologous regenerative therapies. Veterinary experience with short-term (MSCs prepared after the injury has occurred) cryopreserved MSCs in naturally occurring injury in the horse will be of value to human practitioners.
\end{abstract}

Methods: Equine bone marrow derived MSCs were cryopreserved in 6 different solutions consisting of $20 \%$ serum, $10 \%$ DMSO and $70 \%$ media or $95 \%$ serum and $5 \%$ DMSO. Serum was autologous serum, commercially available pooled equine serum or fetal bovine serum (FBS). Cell survival, morphology and growth kinetics were assessed by total cell number, measurement of growth kinetics, colony-forming-unit-assay and morphology of MSCs after monolayer culture post-thaw.

Results: There were no significant differences in post-thaw viability, total cell number, morphology scores or growth kinetics among the 6 solutions. Post thaw viabilities from each group ranged from $80-90 \%$. In all solutions, there were significantly fewer MSCs and the majority (99\%) of MSCs remained in the original generation 24 hours post-thaw. Seventy two hours post-thaw, the majority of MSCs (50 \%) were proliferating in the fourth generation. Mean colony count in the CFU-F assay ranged from 72 to 115 colonies.

Conclusions: Each of the serum sources could be used for short-term cryopreservation of equine bone marrow derived MSCs. Prior to clinical use, clinicians may prefer autologous serum and a lower concentration of DMSO.

Keywords: Mesenchymal stem cell, Cryopreservation, Fetal bovine serum, Serum, Equine

\footnotetext{
* Correspondence: awatts@crm.tamu.edu

'Department of Large Animal Clinical Sciences, Texas A\&M University,

College Station, TX 77843, USA

Full list of author information is available at the end of the article
}

C Biomed Central

(c) 2015 Mitchell et al. Open Access This article is distributed under the terms of the Creative Commons Attribution 4.0 International License (http://creativecommons.org/licenses/by/4.0/), which permits unrestricted use, distribution, and reproduction in any medium, provided you give appropriate credit to the original author(s) and the source, provide a link to the Creative Commons license, and indicate if changes were made. The Creative Commons Public Domain Dedication waiver (http://creativecommons.org/publicdomain/zero/1.0/) applies to the data made available in this article, unless otherwise stated. 


\section{Introduction}

The equine athlete is a well-accepted model for stem cell therapies in musculoskeletal injury [1]. This is because the horse suffers from naturally occurring superficial digital flexor tendon injury that is similar to humans, and culture-derived and expanded mesenchymal stem cells (MSCs) are being used to treat these injuries [2]. Use of clinical practices in equine cellular therapies that are acceptable in human medicine would be beneficial to help ascertain the value of stem cell therapy for tendon injury in this naturally occurring large animal model.

The ideal stem cell preparation, whether frozen or fresh, is an ongoing debate in medicine [3-6]. Cryopreserved MSCs are used in approximately $35 \%$ of published MSC clinical trials [7]. However, in veterinary medicine the majority of laboratories preparing MSCs for horses throughout the world do so with fresh cells [8]. This is not to state that MSCs have not been previously frozen, but that immediately prior to implantation in the patient the MSCs are in monolayer culture and are prepared for injection immediately prior to clinical use with transport to the animal site in cooled media. Identification of a cryopreservation medium that allows for immediate clinical use of MSCs post thaw would be beneficial to streamline laboratory and clinical procedures and reduce associated costs. It is also possible that the cryopreservation process itself induces cell selection of "stronger" MSCs or induces greater MSC activity and expansion potential, which could translate to improved stem cell efficacy [9].

Because of the potential benefits of using cryopreserved MSCs, and the use of cryopreserved MSCs in human clinical trials, cryopreserved MSCs should be investigated in the treatment of naturally occurring tendon injury in horses. The first step in using cryopreserved MSCs in equine veterinary patients is to identify the ideal medium for cryopreservation. To do this, the effect on short-term viability and growth of MSCs post thaw must be understood [10]. Our objective was to determine whether a clinically acceptable formulation and serum source for short-term cryopreservation of equine bone marrowderived MSCs would preserve normal viability, morphology, and normal growth kinetics post thaw. Six different freezing solutions were tested with differing serum supplementation sources and concentrations of dimethyl sulfoxide (DMSO). Different DMSO formulations were tested to determine whether a low concentration of DMSO was sufficient to preserve viability and growth of MSCs frozen in a slow-freezing method. Different serum sources were tested to determine whether an autologous serum source was sufficient to preserve viability and growth. We hypothesized that there would be no differences in the postthaw viability, morphology, and cell growth kinetics in MSCs cryopreserved in autologous, allogeneic, or xenogeneic media or with different concentrations of DMSO.

\section{Methods}

Bone marrow-derived MSC isolation, expansion, and cryopreservation

All animal procedures were approved by the institution's animal care and use committee (IACUC 2012-079). No horses were euthanized for this study. Bone marrowderived MSCs were isolated from nine healthy horses ranging in age from 5 to 16 as described previously [11]. Briefly, bone marrow was collected from mildly sedated horses into heparinized syringes for a final concentration of 5000 units of heparin per $30 \mathrm{ml}$ marrow. Red blood cell lysis was performed with ammonium chloride $(7.7 \mathrm{mg} / \mathrm{ml}$ $\mathrm{NH}_{4} \mathrm{Cl} ; 2.06 \mathrm{mg} / \mathrm{ml}$ hydroxymethane-aminomethane; $\mathrm{pH}$ 7.2) [11]. The remaining nucleated cellular portion of the marrow was plated at $175 \mu$ l original raw marrow volume $/ \mathrm{cm}^{2}$ (Corning, Corning, NY, USA) and maintained at $37{ }^{\circ} \mathrm{C}, 5 \% \mathrm{CO}_{2}$ in humidified air. Culture medium (Dulbecco's modified Eagle's medium $1 \mathrm{~g} / \mathrm{l}$ glucose (Mediatech, Manassas, VA, USA) supplemented with $10 \%$ fetal bovine serum (FBS) (HyClone Inc., Logan, UT, USA), $2.5 \%$ HEPES buffer (Corning), and 10,000 units/ml penicillin, $10,000 \mu \mathrm{g} / \mathrm{ml}$ streptomycin, $25 \mu \mathrm{g} / \mathrm{ml}$ amphotericin B (Life Technologies, Grand Island, NY, USA)) was exchanged three times per week. Once colonies or monolayers reached $70 \%$ of confluence, cultures were passaged until there was a minimum number of MSCs available for the experiment of $3 \times 10^{6} \mathrm{MSCs}$ and for other experiments not outlined in this manuscript. During each passage, cells were detached from culture flasks by incubation with $5 \mathrm{ml}$ per $175 \mathrm{~cm}^{2}$ of $0.25 \%$ trypsin-ethylenediamine tetraacetic acid (EDTA) (Corning) for 5 minutes followed by collection, serum neutralization of trypsin with $5 \mathrm{ml}$ per $175 \mathrm{~cm}^{2}$ of $10 \%$ equine serum in Hank's balanced salt solution (HBSS), and centrifugation. MSCs were resuspended in culture medium and reseeded into new tissue culture flasks at $5000 \mathrm{MSCs} / \mathrm{cm}^{2}$. Once the minimum total MSC number was achieved, MSCs were collected, rinsed by centrifugation three times, and a portion frozen in each cryopreservation solution at $10 \times 10^{6} \mathrm{MSCs} / \mathrm{ml}$. Freezing media were a formulation of $20 \%$ serum, $10 \%$ DMSO, and $70 \%$ minimum essential media (MEM) $(20 / 10 / 70)$ or a formulation of $95 \%$ serum and $5 \%$ DMSO (95/5). Serum sources were FBS, commercially available pooled equine serum (Thermo Scientific, Waltham, MA, USA), or autologous serum (FBS, allogenic, autologous). The same lot was used throughout the project for both FBS and pooled equine serum. After dropwise addition to the freezing solution, the cell suspension was transferred to cryovials (Thermo Scientific) which were placed immediately in a room-temperature isopropyl alcohol freeze container (Thermo Scientific), and stored in a $-80{ }^{\circ} \mathrm{C}$ freezer for 24 hours. After 24 hours, the vials were transferred to liquid nitrogen storage in the liquid phase [12]. 


\section{Immunophenotyping}

MSCs grown for each horse underwent immunophenotyping for expression of MHCII (Bio-Rad, Raleigh, NC, USA), CD44 (Bio-Rad), CD29 (Beckman Coulter, Brea, CA, USA), CD90 (VMRD Inc., Pullman, WA, USA), and CD45RB (VMRD Inc.) using flow cytometry. Antibodies were chosen based on previously published work and dilution of 1:400 for CD90, 1:10 for CD45RB, and 1:100 for CD44, CD29, and MHCII [13, 14]. MSCs were aliquoted at 1 million cells per Eppendorf tubes in $50 \mu \mathrm{l}$ staining buffer.

MSCs stained with primary antibodies (MHCII, CD29, and CD44) had antibody dilution added and were incubated for 45 minutes at $4{ }^{\circ} \mathrm{C}$. Pellets were centrifuged $(400 \times$ g, 7 brake) for 5 minutes. Pellets were washed with $200 \mu$ l Dulbecco's phosphate-buffered saline (DPBS; Lonza, Walkersville, MD, USA) and centrifuged again before being resuspended in DPBS for analysis.

MSCs stained with secondary antibodies (CD90, CD45RB) had antibody dilution added and cells were incubated on ice for 15 minutes before being centrifuged $\left(400 \mathrm{~g}, 4{ }^{\circ} \mathrm{C}\right.$ ) for 3 minutes. Cells were washed with $100 \mu \mathrm{l}$ DPBS and centrifuged twice. One hundred microliters of secondary antibody dilution (1:100) was added and cells were incubated on ice in the dark for 15 minutes before being centrifuged $(400 \times g)$ for 5 minutes. Cells were washed with $200 \mu \mathrm{l}$ DPBS and centrifuged again before being resuspended in $500 \mu \mathrm{l}$ DPBS for analysis. Both primary and secondary antibodies had $5 \mu \mathrm{l}$ 7-AAD (Biolegend, San Diego, CA, USA) added immediately prior to analysis for assessment of viability.

\section{Trilineage differentiation}

Multipotency of MSCs used in the experiment was assessed by inducing trilineage differentiation on MSCs from eight of nine horses using techniques described previously [15-17]. For chondrogenic differentiation, three aliquots of 500,000 MSCs were centrifuged $(300 \times g$, 5 minutes, $4{ }^{\circ} \mathrm{C}, 7$ brake) in polypropylene conical tubes to form pellets. Supernatant was aspirated gently not to disturb the pellet and $1 \mathrm{ml}$ chondrogenic media was added. Chondrogenic media containing Dulbecco's modified Eagle's medium $4.5 \mathrm{~g} / \mathrm{l}$ glucose supplemented with $1 \%$ FBS, $2.5 \%$ hepes buffer, 10,000 units $/ \mathrm{ml}$ penicillin, $10,000 \mu \mathrm{g} / \mathrm{ml}$ streptomycin, $25 \mu \mathrm{g} / \mathrm{ml}$ amphotericin B, $0.2 \%$ transforming growth factor beta (Life Technologies, Grand Island, NY, USA), $301.89 \mu \mathrm{g}$ dexamethasone (Sigma Aldrich, St. Louis, MO, USA), $50 \mu \mathrm{g} / \mathrm{ml} \mathrm{L}$-ascorbic acid (Sigma Aldrich), $40 \mu \mathrm{g} / \mathrm{ml}$ proline (Sigma Aldrich), and $1 \%$ ITS premix (VWR, Radnor, PA, USA) were exchanged three times per week for 21 days. Pellets were fixed in $4 \%$ PFA (Sigma Aldrich) for 10 minutes followed by routine embedding, sectioning, and staining with toluidine blue (Sigma Aldrich).
For adipogenic differentiation, MSCs were seeded to $10 \mathrm{~cm}$ plates at $1000 \mathrm{MSCs} / \mathrm{cm}^{2}$. Once the plates reached $70 \%$ confluence, media were exchanged for adipogenic induction media for 3 days (Dulbecco's modified Eagle's medium F12 (VWR) supplemented with 3 \% FBS, 10,000 units $/ \mathrm{ml}$ penicillin, $10,000 \mu \mathrm{g} / \mathrm{ml}$ streptomycin, $25 \mu \mathrm{g} / \mathrm{ml}$ amphotericin B, $5 \%$ rabbit serum (Life Technologies), $33 \mu \mathrm{M} / \mathrm{l}$ biotin (Sigma Aldrich), $17 \mu \mathrm{M} / \mathrm{l}$ pantothenate (Sigma Aldrich), $1 \mu \mathrm{M} / 1$ insulin (Sigma Aldrich), $1 \mu \mathrm{M} / \mathrm{l}$ dexamethasone, $225 \mu \mathrm{l}$ isobutylmethylxanthine (Sigma Aldrich), $89 \mu \mathrm{l}$ rosiglitazone (Sigma Aldrich)). Media were then exchanged for adipogenic maintenance media for an additional 3 days (adipogenic induction media without isobutylmethylxanthine and rosiglitazone). Induced and control plates were stained with Oil Red O (Sigma Aldrich).

For osteogenic differentiation, MSCs were seeded to $10 \mathrm{~cm}$ plates at $1000 \mathrm{MSCs} / \mathrm{cm}^{2}$. After reaching $70 \%$ confluence, media were exchanged for osteogenic induction medium and maintained for 14 and 21 days (Dulbecco's modified Eagle's medium F12 supplemented with $10 \%$ FBS, 10,000 units $/ \mathrm{ml}$ penicillin, 10,000 $\mu \mathrm{g} / \mathrm{ml}$ streptomycin, $25 \mu \mathrm{g} / \mathrm{ml}$ amphotericin B, $10 \mu \mathrm{M} / \mathrm{l} \beta$-glycerophosphate (Sigma Aldrich), $20 \mathrm{nM} / \mathrm{l}$ dexamethasone, and $50 \mu \mathrm{g} / \mathrm{ml}$ L-ascorbic acid). Plates were stained with $2 \%$ Alizarin Red (Sigma Aldrich).

\section{Thawing}

After storage in liquid nitrogen for $2-5$ days, vials were thawed with gentle agitation at $35{ }^{\circ} \mathrm{C}$ in a water bath until an ice ball was no longer present. Immediately post thaw, an equal volume of DPBS was added to the cell suspension. Five minutes later, the cell suspension was collected and added dropwise to $20 \mathrm{ml}$ DPBS (Lonza). This thawing method was defined in a pilot project to this experiment where we determined the importance of avoiding osmotic shock in the 95/5 formulations (Additional file 1). A $100 \mu \mathrm{l}$ aliquot of thawed cell suspension in DPBS was used for a total cell count and viability using fluorescein diacetate $(67.57 \mathrm{mg} / \mathrm{ml})$ and propidium iodide $(1.35 \mathrm{mg} / \mathrm{ml})$ in DPBS. Counting cell suspensions were plated on a Nebauer hemocytometer and visualized by fluorescence microscopy (Olympus, Center Valley, PA, USA). The live (green) and dead (red) cells were counted. A total of 10 squares were counted per sample. The cell suspension was carefully mixed by pipetting and then centrifuged to pellet the cells for removal of freezing solutions (300 $\times g, 5$ minutes, $4{ }^{\circ} \mathrm{C}, 7$ brake).

\section{Post-thaw cell staining with CellTrace ${ }^{\mathrm{TM}}$ label}

Cell Trace ${ }^{\mathrm{rm}}$ violet dye was used to determine the speed of cellular proliferation. CellTrace ${ }^{\mathrm{Tm}}$ violet dye binds to intracellular amines without interfering with cellular activity. As the cell divides, the dye is distributed equally 
between the two daughter cells, resulting in dye dilution that reflects the number of cell divisions which have occurred since labeling $[18,19]$ with CellTrace ${ }^{\mathrm{Tm}}$ (Life Technologies, ThermoFisher Scientific, Grand Island, NY 14072). The amount of dye dilution in each cell and thus the number of cell divisions which have occurred, or the generation of that cell, is determined using flow cytometry. A cell that has dye dilution reflecting two cellular divisions is a second-generation cell, and so on. Post centrifugation, the supernatant was removed and pelleted MSCs were resuspended in $1 \mathrm{ml}$ DPBS to be labeled with CellTrace ${ }^{\mathrm{Tx}}$ violet dye, as per the manufacturer's instructions. Briefly, cells were labeled in suspension by adding $1 \mu \mathrm{l}$ staining solution to $1 \mathrm{ml}$ DPBS containing $\leq 10 \times 10^{6}$ cells. CellTrace ${ }^{\mathrm{Tx}}$ violet dye and MSCs were incubated for 20 minutes at room temperature in the dark with gentle agitation every $3 \mathrm{mi}-$ nutes. Complete media were added at five times the staining volume and incubated at room temperature in the dark for an additional 5 minutes. The suspension was centrifuged, resuspended in complete media at the same volume, and incubated for another 10 minutes at room temperature in the dark, with agitation every $3 \mathrm{mi}-$ nutes. Stained MSCs were then treated as described in the following for post-thaw monolayer culture of MSCs. Life Technologies, ThermoFisher Scientific, Grand Island, NY 14072.

\section{Post-thaw monolayer culture of MSCs}

Following labeling, MSCs from each condition were seeded at 10,000 viable MSCs $/ \mathrm{cm}^{2}$ to tissue culture flasks (Corning) to evaluate growth kinetics, viability, and morphology and 1,000 viable MSCs were seeded to a $10 \mathrm{~cm}$ plate for colony-forming unit assay. Cultures were maintained as already outlined prior to cryopreservation.

Monolayer cultures of MSCs were visualized by microscopy and photographed (Olympus) using commercially available software (cellSens; Olympus). Each monolayer culture was given a morphology score of excellent (cells were spindle shaped), good (cells were wider or more star shaped), fair (cells were flattened and/or contained large vacuoles), or poor (cells were flattened, vacuolar, and foamy in appearance) and a debris score of none ( $<5$ floating cells per $40 \times$ field), mild ( $<20$ floating cells per $40 \times$ field floating cells), moderate ( $<40$ floating cells per $40 \times$ field), or severe ( $\geq 40$ floating cells per $40 \times$ field) by an investigator blinded to treatment group assignment (Table 1). One week after seeding the $10 \mathrm{~cm}$ plates, colonies were stained with $3 \%$ crystal violet (Sigma Aldrich) and colonies were manually counted without magnification. The evaluator was masked to treatment group assignment.

\section{Cell generation assay}

After 24 and 72 hours of monolayer culture post thaw, cells were detached and collected by addition of trypsinEDTA. The total cell number was determined. Cells were colabeled with propidium iodide and flow cytometry was used to assess the concentration of remaining CellTrace ${ }^{\mathrm{Tu}}$ cytoplasmic dye. Approximately 12,000-35,000 events were collected per condition. The cell generation with the greatest concentration of cytoplasmic dye at 24 hours post thaw was defined as the parent generation. The ModFit LT software program (Verity Software House, Topsham, ME, USA) was used to determine division rates of the MSCs. Results were reported as the current cell generation and the proportion of cells in that generation.

\section{Statistical analysis}

Raw data were imported to a commercial statistical software program (Statistix 9; Analytical Software, Tallahassee, FL, USA). Differences between the conditions for continuous data were evaluated by one-way analysis of variance (ANOVA) with Tukey's post-hoc tests and by KruskalWallis ANOVA with pairwise comparisons as appropriate for the data structure. Differences in paired data within a group were evaluated by the Wilcoxon signed-rank test. Differences were considered significant when $p \leq 0.05$.

\section{Results}

No differences were found in the post-thaw viability, morphology, and growth kinetics of previously frozen MSCs with each of the tested solutions. Bone marrow was collected from nine mixed-breed mares aged 5-16 years. The passage number of MSCs ranged from passage 3 to passage 6 (Table 2). Differences in passage number were due to the need for different total numbers of MSCs from each horse for other experiments not outlined in this manuscript.

All assay time points were met for each donor and formulation except for Horse 6 in 95/5FBS, due to a laboratory error immediately after CellTrace ${ }^{\mathrm{Tm}}$ labeling. This freezing medium (95/5FBS) was repeated later and all

Table 1 Age of mesenchymal stem cell donors and passage number

\begin{tabular}{lll}
\hline & Morphology & Debris \\
\hline Excellent/none & Cells were spindle shaped & $<5$ floating cells per 40x field \\
Good/mild & Cells were wider or more star shaped & $<20$ floating cells per 40x field \\
Fair/moderate & Cells were flattened and/or contained large vacuoles & $<40$ floating cells per 40x field \\
Poor/severe & Cells were flattened, vacuolar, and foamy in appearance & $\geq 40$ floating cells per 40x field \\
\hline
\end{tabular}


Table 2 Morphology and debris scoring system

\begin{tabular}{lll}
\hline Horse & Age & Passage \\
\hline 1 & 8 & 4 \\
2 & 5 & 6 \\
3 & 16 & 4 \\
4 & 12 & 4 \\
5 & 11 & 3 \\
6 & 7 & 4 \\
7 & 13 & 6 \\
8 & 12 & 3 \\
9 & 14 & 6 \\
\hline
\end{tabular}

assays were performed. For repetition of Horse 6 95/5FBS, the same passage was available but had been cryopreserved for 18 months. Statistical significance was unchanged with the repeated data for Horse 6 in 95/5 FBS.

Within each solution, there were significantly fewer viable and attached MSCs at 24 hours post thaw compared with the number of viable MSCs seeded to each flask (Fig. 1; 20/10/70FBS, $p=0.02$; 20/10/70Allo, $p=0.05$; 20/ 10/70Auto, $p=0.012$; 95/5FBS, $p=0.05$; 95/5Allo, $p=0.05$; 95/5Auto, $p=0.02$ ).

Between the solutions there were no significant differences in any of our assays. Immediate post-thaw cell viability for each condition ranged from 81 to 88 \% (Fig. 2). Cell debris scores at 24 hours were generally mild to moderate debris, with one or two horses in each medium having severe debris and one or two horses in each medium with no debris (Fig. 3). MSCs receiving severe debris scores were from the same two individual donors. Horse 1 MSCs received a debris score of severe for all six freezing solutions and the total viable MSCs at 24 hours were extremely low, ranging from 25,000 to 55,000 viable MSCs. MSCs from one other individual donor (Horse 6) received severe debris scores in two formulations, 20/10/70FBS and 95/5Auto, and scores of mild and moderate for all other formulations. The cell counts at 24 hours were also very low for these formulations (145,000 and 45,000 MSCs). In contrast to scores at 24 hours, cell debris scores at 72 hours were generally none to mild (Fig. 3). Exceptions to this were the MSCs from the same two individual horses that had received severe debris scores at 24 hours. The MSCs from these horses also received worse scores at 72 hours: Horse 1 had moderate debris in all formulations except 20/10/ 70FBS and 20/10/70Auto, and Horse 6 had severe debris in 95/5Auto. Cell morphology scores at both 24 and 72 hours post thaw were generally good to excellent without differences between groups (Figs 3 and 4). Total viable cell counts were not different between the groups at 24 or 72 hours (Fig. 1). The percentage of confluence at 72 hours ranged from 70 to $80 \%$ for all conditions. Numbers of colonies from the $10 \mathrm{~cm}$ plates ranged from 72 to 115 colonies (Fig. 5). At 24 hours post thaw, the majority (mean; standard deviation) of MSCs remained in their parent generation: 20/10/70FBS (98.4\%; 3.15), 20/10/70Allo (98.2\%; 2.36), 20/10/70Auto (99.5\%; 0.44), 95/5FBS (98.8 \%; 1.48), 95/5Allo (98.6\%; 1.92), and 95/5Auto (98 \%; 2.96) (Fig. 6 and Additional file 2). At 72 hours post thaw, the majority (mean; standard deviation) of MSCs were in the fourth generation: 20/10/70FBS (54.9 \%; 10.25), 20/10/ 70Allo (55.1 \%; 7.94), 20/10/70Auto (57 \%; 10.32), 95/5FBS (51.5\%; 15.17), 95/5Allo (54.2\%; 12.98), and 95/5Auto (59.1\%; 11.78) (Fig. 6 and Additional file 3). When the number of MSCs contributing to the total cell number at
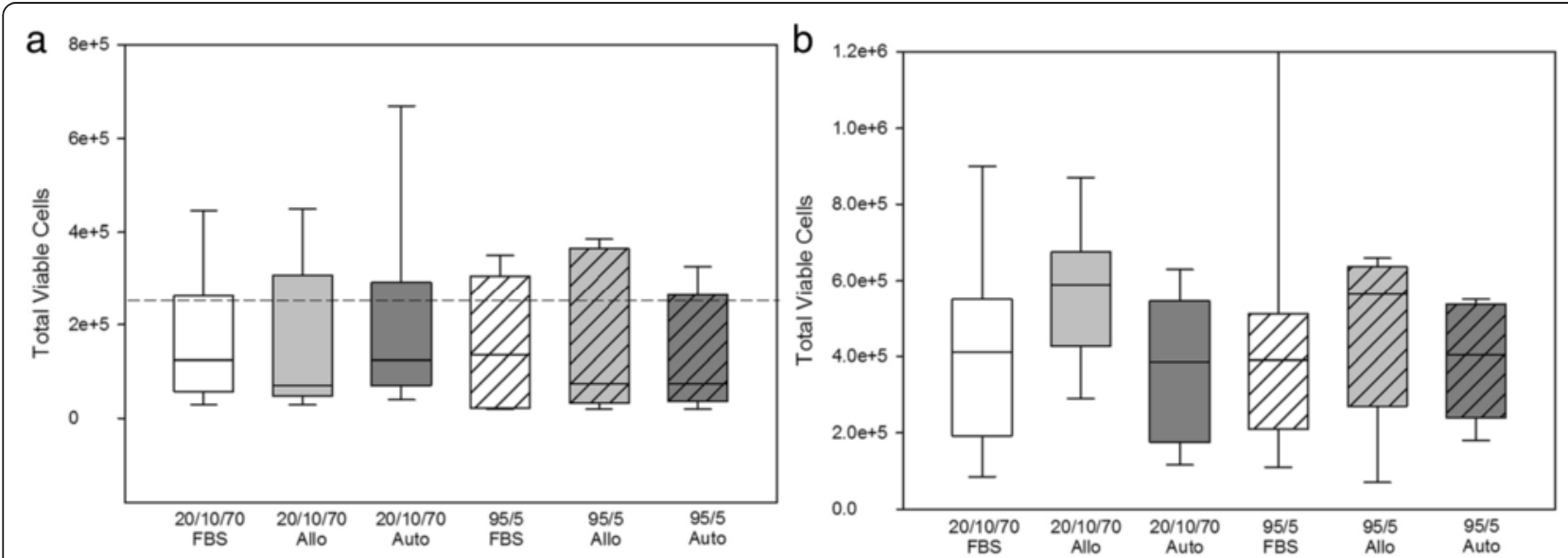

Fig. 1 Total viable cell number. Viability of MSCs from nine horses cryopreserved in six different freezing solutions after $\mathbf{a} 24$ hours and $\mathbf{b} 72$ hours in monolayer culture post thaw (median, quartiles). There were no differences between the groups ( 24 hours, $p=0.96 ; 72$ hours, $p=0.51)$. a Dotted line represents the total number of viable MSCs seeded post thaw. The total viable MSCs at 24 hours post thaw were significantly lower than the cell number seeded in all groups (20/10/70FBS, $p=0.02 ; 20 / 10 / 70 A l l o, p=0.05 ; 20 / 10 / 70$ Auto, $p=0.01 ; 95 / 5 F B S, p=0.05 ; 95 / 5$ Allo, $p=0.05 ; 95 / 5$ Auto, $p=0.02$ ). Allo allogenic, Auto autologous, FBS fetal bovine serum, 20/10/70 $20 \%$ serum, $10 \%$ dimethyl sulfoxide, $70 \%$ minimum essential media, $95 / 5$ 95 \% serum, $5 \%$ dimethyl sulfoxide 


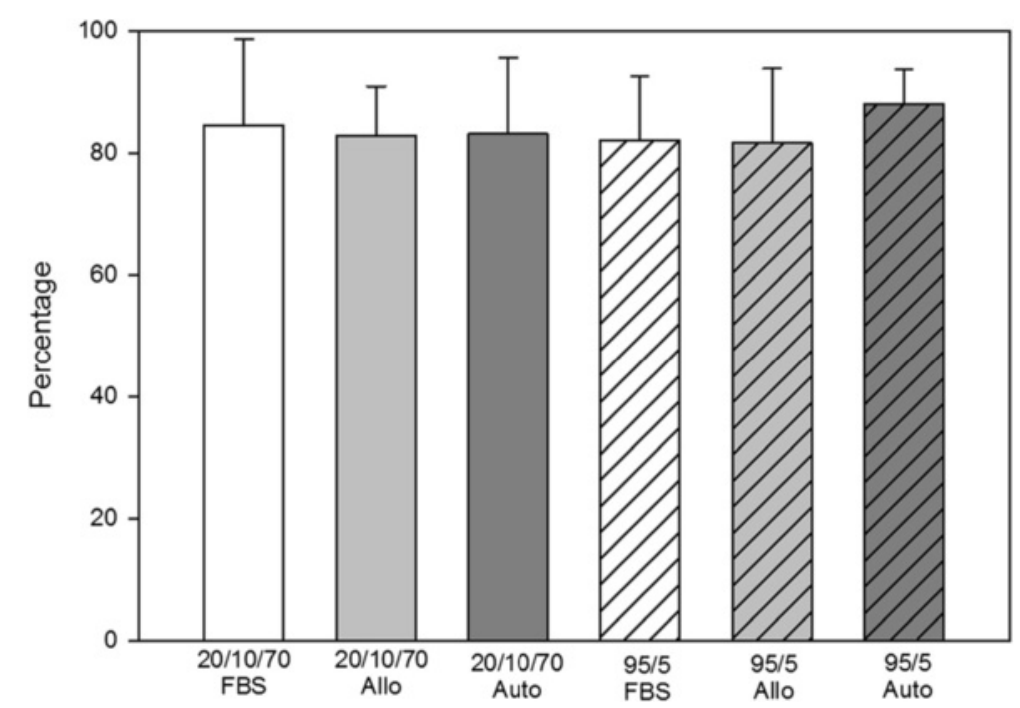

Fig. 2 Percentage of viable cells post thaw. Viability immediately post thaw of MSCs from nine horses cryopreserved in six different solutions (mean; standard deviation). There were no significant differences between the groups. $p=0.844$. Allo allogenic, Auto autologous, FBS fetal bovine serum, 20/10/70 $20 \%$ serum, $10 \%$ dimethyl sulfoxide, $70 \%$ minimum essential media, 95/5 95\% serum, $5 \%$ dimethyl sulfoxide

72 hours was calculated, based upon the mean proportion of cells in each generation at 72 hours, it was lower than the cell count at 24 hours (20/10/70FBS, 65,372; 20/10/ 70Allo, 95,865; 20/10/70Auto, 60,675; 95/5FBS, 80,897; 95/ 5Allo, 80,033; 95/5Auto, 64,578).

The majority of horses were negative for MHCII, CD44, and CD45RB and were positive for CD29 and CD90. Eight of nine horses underwent trilineage differentiation and were positive for osteogenic, chondrogenic, and adipogenic differentiation (Table 3; Fig. 7).

\section{Discussion}

We sought to identify whether a clinically acceptable medium for short-term cryopreservation of equine bone marrow-derived MSCs would preserve normal post-thaw viability and growth. MSCs from nine middle-aged adult horses at a broad range of passage numbers were utilized to best mimic the clinical scenario of autologous MSC therapy where differing total numbers of MSCs might be required due to differences in tendon lesion size and severity. Varying concentrations of autologous serum, pooled equine serum, or FBS; two concentrations of DMSO; and the presence or absence of a cell culture media were tested. Standard immediate and longer-term post-thaw viability assessments included total live and dead analysis, colony forming units-fibroblast (CFU-F) assay, and assessment of MSC morphology and cellular debris. A more novel analysis we used to assess growth was to stain MSC cytoplasm in a way that would not interfere with cellular activity and could be accurately measured by flow cytometry, giving us the number and frequency of cellular divisions for single cells [20].
Analysis of remaining cytoplasmic dye 24 and 72 hours after staining allowed evaluation of growth kinetics of MSCs from each cryopreservation medium, and in combination with total cell numbers and culture scoring enabled indirect assessment of post-thaw apoptosis induction.

One of the benefits of studying stem cell therapies in the horse is that the horse population, like that of man, is not homogeneous in genotype or phenotype, unlike most laboratory species. In addition to genotype and phenotype differences, individual variation in MSC characteristics, especially in species with diversity, has been reported $[14,21]$. It is important to assess MSCs in models that more accurately reflect the inherent variability among human MSC preparations. Utilizing a greater number of individuals in MSC experiments better reflects responses from a diverse population. Using MSCs from nine individual donors, we found no differences between any of the freezing medium formulations in the post-thaw viability or early growth and morphology of MSCs by any of our assay methods. However, when we looked at individual horses, there were marked differences in cell expansion between the media solutions 72 hours post thaw in a few of the horses. For example, $37 \%$ of MSCs from Horse 4 frozen in 20/10/70Allo were in generation 5 , while the other five freezing solutions were much lower, ranging from 12 to $31 \%$ of the MSC population in generation 5. As a contrasting example, only $0.5 \%$ of MSCs from Horse 6 frozen in 20/10/ 70Allo had reached generation 5, while the other five freezing media had much higher percentages of MSCs in generation 5, ranging from 6 to $62 \%$. Had we included 


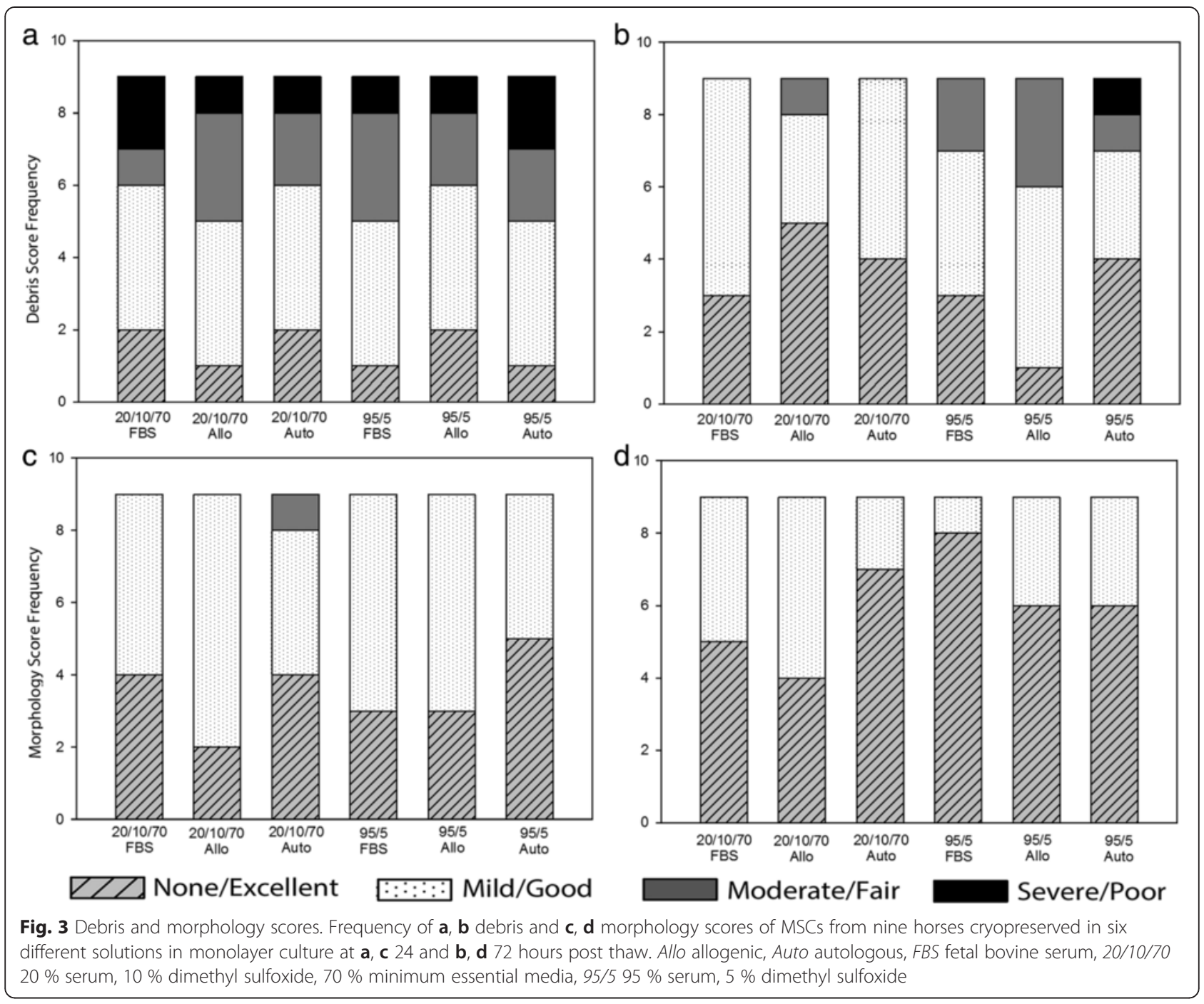

one of these horses in a smaller group size, we might have erroneously identified differences between the formulations.

The media formulations we tested were either $20 \%$ serum, $10 \%$ DMSO, and $70 \%$ cell culture media or $95 \%$ serum and $5 \%$ DMSO. The 20/10/70 formulation was elected as the standard cryopreservation medium formulation used in cell culture for many cell types. Within this group, our question was whether use of xenogen-free serum sources were possible. The $95 / 5$ formulation that has been reported recently was elected to answer two questions [22]: can an almost entirely autologous product (95\%) and a reduced DMSO concentration be used? The lack of deleterious effects when an autologous product was used with a low concentration of DMSO could move cryopreserved MSCs closer to an off-the-shelf product and would also streamline preparation of autologous MSCs.
Culture and cryopreservation of MSCs in FBS has been a standard technique for many years. Because of a desire to move toward an entirely xenogen-free product in stem cell therapies, two equine serum sources were tested. Based upon other work in our laboratory (data not shown) and that of others $[7,23]$, we think there are individual differences in the quality of serum for the growth of MSCs. Because of these potential variations in serum quality between individual horses, autologous serum and a commercially available pooled equine serum were tested. If individual serum quality differences exist, they do not appear to negatively affect the post-thaw viability and growth of MSCs frozen in autologous serum at either concentration we report here. Therefore, either the commercially available equine serum or autologous serum can be used for short-term xenogen-free MSC cryopreservation. An entirely autologous product versus an allogeneic, xenogen-free product 


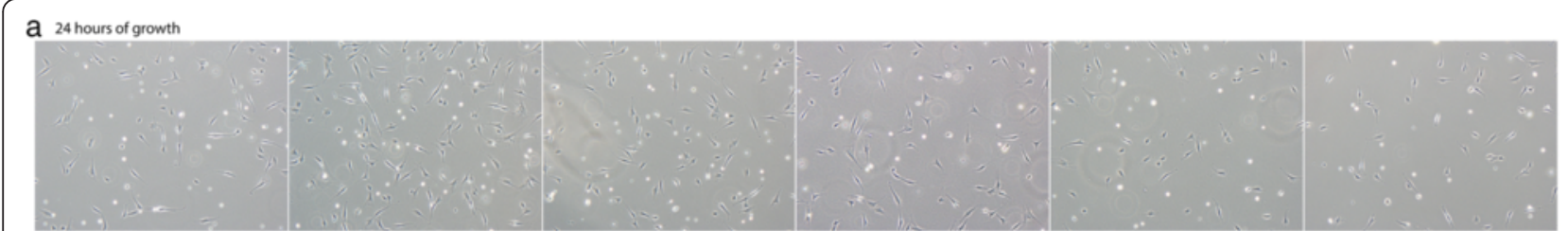

b 72 hours of growth

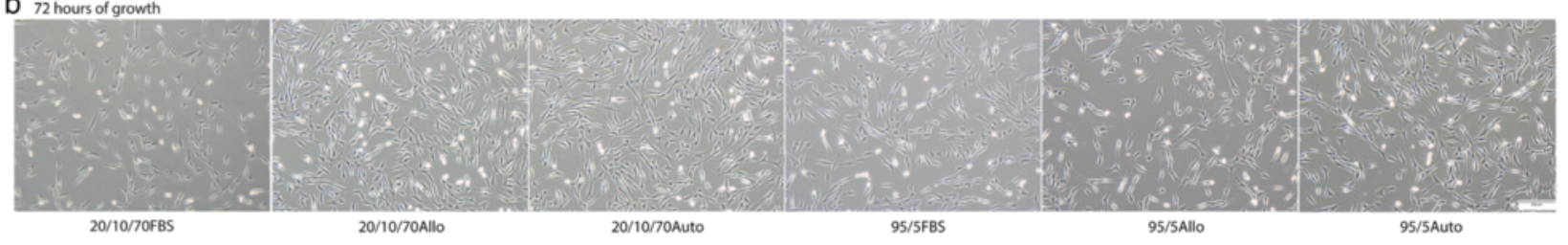

Fig. 4 Images of monolayer culture. Microscopy images of MSCs from Horse 3 cryopreserved in six different freezing solutions after a 24 hours and $\mathbf{b} 72$ hours in monolayer culture post thaw. Original magnification 40x, scale bar $=500 \mu$ m. Allo allogenic, Auto autologous, FBS fetal bovine serum, 20/10/70 $20 \%$ serum, $10 \%$ dimethyl sulfoxide, $70 \%$ minimum essential media, 95/5 95\% serum, $5 \%$ dimethyl sulfoxide

would be desirable to minimize many more risks, both known and unknown.

Despite being cytotoxic and potentially toxic to the patient who will receive the cells, $10 \%$ DMSO is the most commonly used cryoprotectant agent with or without cell washing for DMSO removal prior to cell infusion to patients $[6,24,25]$. Because of its cytotoxicity and varying reports of the effectiveness of lower DMSO concentrations in human cell cryopreservation, $5 \%$ DMSO was also tested [26, 27]. A lower DMSO concentration, if effective, might minimize toxic effects that occur prior to freezing and in the immediate post-thaw period when MSCs are in the cryopreservation medium. Based upon our results, $5 \%$ DMSO is sufficient as a cryoprotectant for short-term MSC cryopreservation. Using this lower concentration of DMSO would be especially important if a post-thaw rinse of MSCs was delayed or avoided altogether prior to clinical application.

Lack of differences among the cryopreservation media we tested is in stark contrast to results of a pilot project in our laboratory. In the pilot project, the same six freezing solutions and serum sources on MSCs from six middle-aged horses were tested, but we utilized a very minor variation in the thawing process. The difference in the thawing method was that post-thaw MSCs were slowly transferred in a dropwise manner to a large volume $(20 \mathrm{ml})$ of DPBS, as has been reported previously, rather than the stepwise introduction to DPBS over 5 minutes we report here [8]. This minor difference in methods resulted in profound deleterious effects of

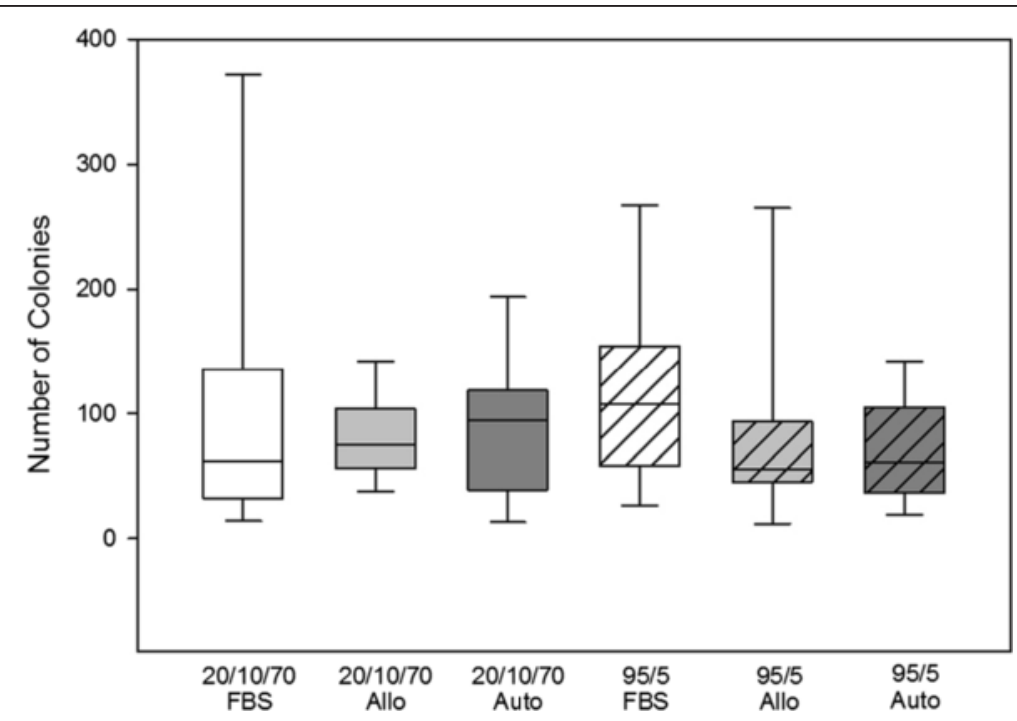

Fig. 5 Total colony number. Numbers of colonies on CFU-F assay from MSCs cryopreserved in six different freezing solutions (median, quartiles). One thousand total viable MSCs were seeded to $10 \mathrm{~cm}$ plates. Colonies were stained and manually counted without magnification 1 week later. There were no differences between the groups. $p=0.76$. Allo allogenic, Auto autologous, FBS fetal bovine serum, 20/10/70 20\% serum, $10 \%$ dimethyl sulfoxide, $70 \%$ minimum essential media, 95/5 $95 \%$ serum, $5 \%$ dimethyl sulfoxide 


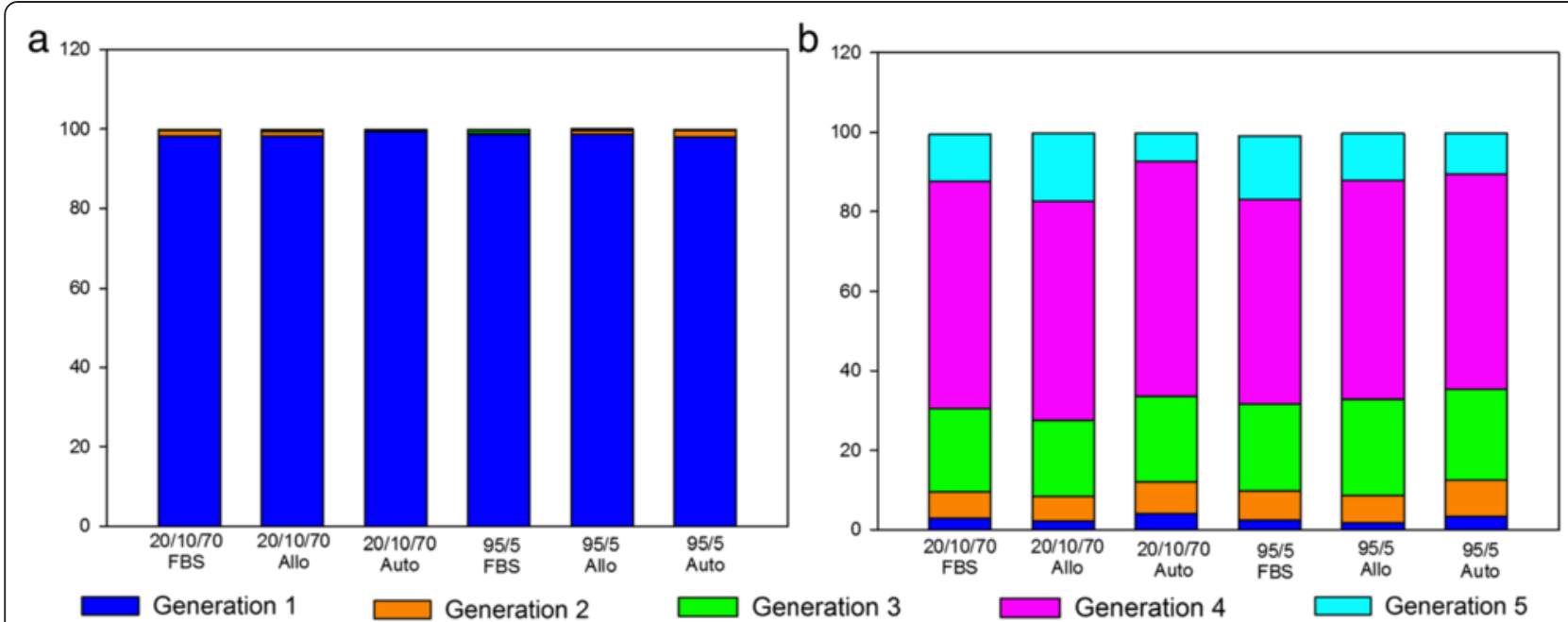

Fig. 6 Cell generations post thaw. Percentage of MSCs cryopreserved in six different solutions in generations $1-5$ at $\mathbf{a} 24$ hours and $\mathbf{b} 72$ hours post thaw and monolayer culture (mean). There were no differences between the groups (generation $2, p=0.82$; generation 3, $p=0.84$; generation $4, p=0.82$; generation 5, $p=0.44$ ), Allo allogenic, Auto autologous, FBS fetal bovine serum, 20/10/70 $20 \%$ serum, $10 \%$ dimethyl sulfoxide, $70 \%$ minimum essential media, 95/5 $95 \%$ serum, $5 \%$ dimethyl sulfoxide

cryopreservation media consisting of $95 \%$ serum of both equine types with post-thaw viabilities of less than $60 \%$ (Additional file 1). Susceptibility of all cell types to postthaw osmotic shock is well known [28] and enhanced susceptibility has been suggested in human MSCs [9]. Both an absence of balanced isotonic solution and/or a lower concentration of cryoprotectant in our 95/5 formulation could have led to increased susceptibility to osmotic shock. Regardless, it appeared in our pilot project that the use of $95 \%$ FBS was somewhat protective of the enhanced susceptibility to osmotic shock in the $95 / 5$ formulation compared with either equine serum source. In the experiment of this report, careful handling of MSCs to reduce osmotic shock resulted in no differences among the $95 / 5$ or $20 / 10 / 70$ formulations. The importance of MSC handling immediately after the thawing process should be underscored.

First reported in 2011, post-thaw growth arrest of MSCs followed by a very rapid proliferation rate of surviving

Table 3 Cell surface marker profiles (percent positive)

\begin{tabular}{llllll}
\hline Horse & MHCII & CD44 & CD29 & CD45 & CD90 \\
\hline 1 & 1.39 & 68.7 & 100 & 7.27 & 100 \\
2 & 1.41 & 3.19 & 100 & 1.56 & 99.8 \\
3 & 1.52 & 1.88 & 99.8 & 2.5 & 83 \\
4 & 1.13 & 71.8 & 100 & 5.52 & 89.7 \\
5 & 1.24 & 10.8 & 99.4 & 14.9 & 94.7 \\
6 & 1.83 & 9.64 & 100 & 6.42 & 99.5 \\
7 & 60.9 & 18.7 & 100 & 39.5 & 92.8 \\
8 & 5.01 & 48.7 & 99.9 & 21.2 & 90.7 \\
9 & 45.4 & 17 & 99.8 & 17.7 & 99.4 \\
\hline
\end{tabular}

MSCs was seen in our study [9]. As originally suggested, this might be selection of "better" MSCs with a younger phenotype and faster proliferation rate while inducing apoptosis of the "less strong" MSCs post thaw. Our study demonstrated a lack of MSC division of the plastic adherent population in the first 24 hours with $>95 \%$ of viable MSCs still in the defined parent generation, and a greater number of nonadherent cells in the first 24 hours post thaw reflected by the higher debris scores at 24 hours and lower total cell count of adherent MSCs after 24 hours of culture than the number of MSCs seeded for all groups. These floating cells were likely apoptotic MSCs, rather than surviving but dysfunctional cells, because much higher numbers and monolayer densities would have occurred at 72 hours had the floating cells recovered function after 24 hours. Additionally, the CFU-F assay colony number was lower when debris scores at 24 hours were high (Fig. 8). This growth arrest seemed to recover between 24 and 72 hours, with the majority of viable MSCs in the fourth generation, 48 hours later. However, we think there was incomplete recovery with continued apoptosis in a portion of MSCs because the total viable cell number at 72 hours was significantly lower than one would expect given our cellular generation data. An assay of apoptosis would have been helpful to prove that apoptosis occurred. Finally, although direct comparisons with growth of MSCs from the same donors that had not been frozen were not made, our impression is that the growth during the 72 hours post thaw was much greater than we see during routine monolayer expansion of fresh MSCs. This is in contrast to a recent report where post-thaw MSC growth was not different to suspension stored MSCs where there was a steady proliferation rate for 4 days [8]. 

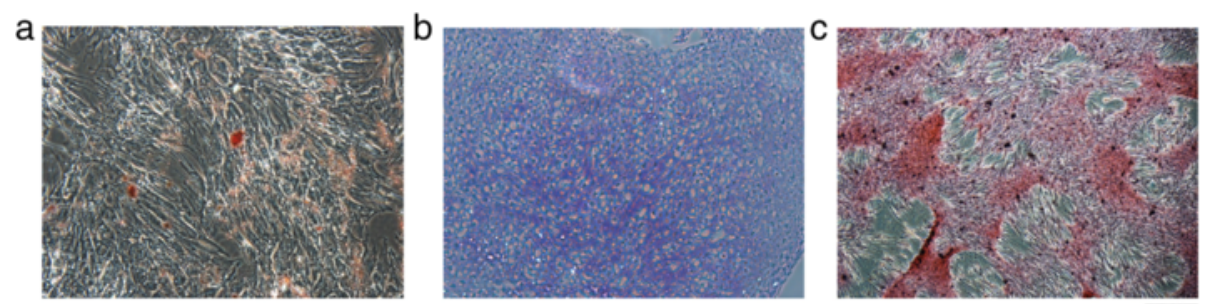

Fig. 7 Trilineage differentiation. Images of MSCs from Horse 6 after a adipogenic, b chondrogenic, and $\mathbf{c}$ osteogenic differentiation. Trilineage differentiation was confirmed on MSCs from eight of the nine horses prior to cryopreservation. One horse did not have MSCs available to be tested. Original magnification 200x, scale bar $=100 \mu \mathrm{m}$

A limitation of our study was that cell surface markers, commonly used to characterize the phenotype of MSCs, and trilineage differentiation potential in vitro were not assayed post thawing. These analyses were not performed for two main reasons. First, others have reported a lack of changes in cell surface markers in fresh versus post-thaw human and porcine bone marrow-derived MSCs and that cryopreservation does not change differentiation ability [9, 29-32]. Second, others have reported lack of changes in cell surface marker profile due to serum type (autologous serum versus FBS) [33]. Therefore, we thought the minimal exposure to different media during freezing and thawing was unlikely to alter the cell surface marker expression or in vitro differentiation potential, so long as viability and growth were unchanged.

Another step that is important to note in our design is that DMSO was removed from MSCs post thaw with a post-thaw wash by centrifugation. In the clinical setting, if one used any of our tested conditions immediately post thaw, a post-thaw wash and centrifugation step would be required if removal of DMSO was desired. This washing step would require laboratory involvement in the clinical procedure, somewhat limiting the off-theshelf availability to the treating clinician.

\section{Conclusion}

We evaluated the short-term cryopreservation of equine bone marrow-derived MSCs in solutions consisting of differing concentrations and types of serum and differing concentrations of DMSO. A low-tech, commercially available freezing system that would be affordable in veterinary services was used. In this system, equine MSCs did not have differences in post-thaw viability and growth, regardless of the cryopreservation formulation or serum source used. The importance of minimizing osmotic shock of MSCs immediately post thaw and the potential increased risk of osmotic shock with different media for

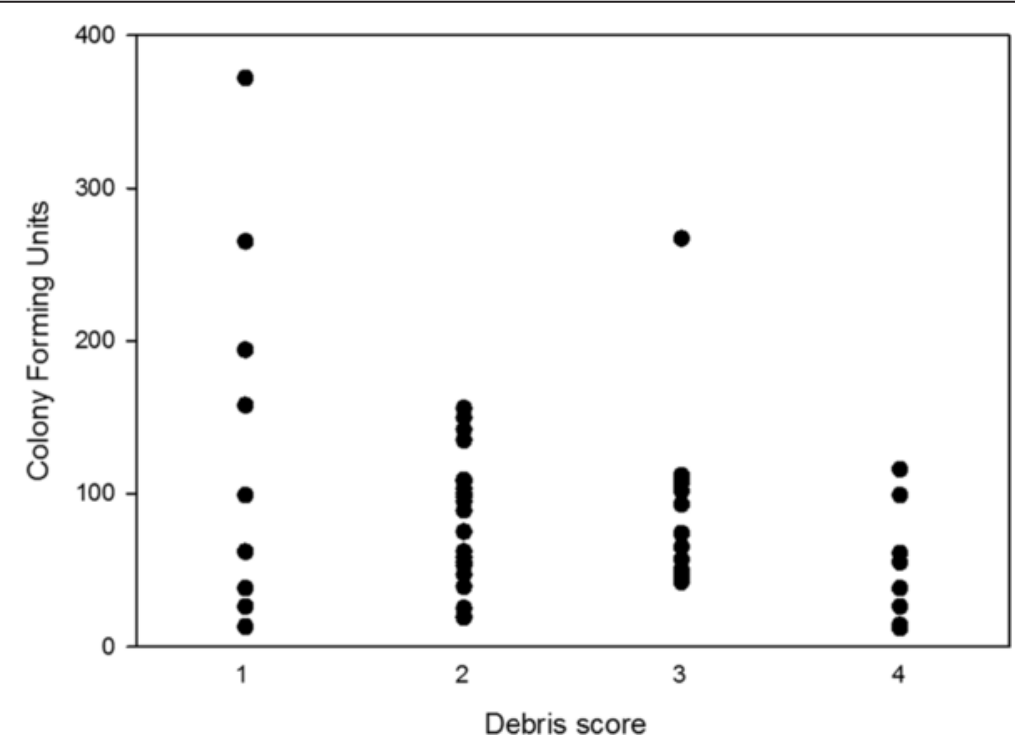

Fig. 8 Debris score versus CFU-F. Total number of colony-forming units plotted against debris scores from MSCs cryopreserved in six different solutions and maintained in monolayer for 24 hours post thaw. When there was increased debris, there were fewer colonies 1 week later, confirming that debris consisted of MSCs which did not recover and adhere to plastic at a later time 
cryopreservation as found in our pilot project should be noted. Additionally, immediately post thaw there was an apparent lag phase of MSCs with little cellular division and assumed apoptosis in the first 24 hours post thaw, followed by rapid MSC growth over the next 48 hours. If a xenogen-free product with lower concentration of cryoprotectant is clinically desirable to streamline clinical and laboratory procedures by use of cryopreserved MSCs, the use of $95 \%$ autologous serum and $5 \%$ DMSO for the short-term cryopreservation of equine bone marrowderived MSCs is recommended.

\section{Additional files}

Additional file 1: Is a figure showing the percentage of viable cells post thaw. Percentage of viable cells post thaw of MSCs from nine horses cryopreserved in six different solutions from our pilot project (median, quartiles). In the pilot project, there was a minor variation in the thawing process. The viable MSCs were significantly lower when MSCs were frozen in 95/5Allo and 95/5Auto solutions. (JPEG $442 \mathrm{~kb}$ )

Additional file 2: Is a figure showing flow histograms of CellTrace ${ }^{\mathrm{TM}}$ dye in MSCs cryopreserved in six different freezing solutions, 24 hours post thaw and monolayer expansion. (JPEG $285 \mathrm{~kb}$ )

Additional file 3: Is a figure showing flow histograms of CellTrace ${ }^{\mathrm{TM}}$ dye in MSCs cryopreserved in six different freezing solutions, 72 hours post thaw and monolayer expansion. (JPEG $1580 \mathrm{~kb}$ )

\section{Abbreviations}

20/10/70: $20 \%$ serum, $10 \%$ dimethyl sulfoxide, 70 \% minimum essential media; 95/5: 95 \% serum, 5 \% dimethyl sulfoxide; ANOVA: Analysis of variance; DMSO: Dimethyl sulfoxide; DPBS: Dulbecco's phosphate-buffered saline; EDTA: Ethylenediamine tetraacetic acid; FBS: Fetal bovine serum; HBSS: Hank's balanced salt solution; MEM: Minimum essential media; MSC: Mesenchymal stem cell.

\section{Competing interests}

The authors declare that they have no competing interests.

\section{Authors' contributions}

AM participated in the study design, performed laboratory work, performed statistical analysis, and drafted the manuscript. KAR and RS participated in the study design, performed laboratory work, and assisted in drafting the manuscript. AEW conceived, designed, and coordinated the study, and assisted in drafting and revising the manuscript. All authors contributed to data interpretation and all authors read and approved the final manuscript.

\section{Acknowledgements}

The authors would like to thank Dr AmandaJo Joswig and Ms Anne Peters for technical assistance. The study was supported by funding from the Link Endowment for Equine Research at Texas A\&M University.

\section{Author details}

${ }^{1}$ Department of Large Animal Clinical Sciences, Texas A\&M University, College Station, TX 77843, USA. ${ }^{2}$ Department of Veterinary Pathobiology, Texas A\&M University, College Station, TX 77843, USA.

Received: 1 April 2015 Revised: 18 September 2015 Accepted: 9 November 2015 Published online: 26 November 2015

\section{References}

1. Smith RK, Garvican ER, Fortier LA. The current "state of play" of regenerative medicine in horses: what the horse can tell the human. Regen Med. 2014;9: 673-85.

2. Smith RK, Korda M, Blunn GW, Goodship AE. Isolation and implantation of autologous equine mesenchymal stem cells from bone marrow into the superficial digital flexor tendon as a potential novel treatment. Equine Vet J. 2003;35:99-102.

3. Kim DH, Jamal N, Saragosa R, Loach D, Wright J, Gupta V, et al. Similar outcomes of cryopreserved allogeneic peripheral stem cell transplants (PBSCT) compared to fresh allografts. Biol Blood Marrow Transplant. 2007;13:1233-43.

4. Eckardt JR, Roodman GD, Boldt DH, Clark GM, Alvarez R, Page C, et al. Comparison of engraftment and acute GVHD in patients undergoing cryopreserved or fresh allogeneic BMT. Bone Marrow Transplant. 1993;11:125-31.

5. Frey NV, Lazarus HM, Goldstein SC. Has allogeneic stem cell cryopreservation been given the "cold shoulder"? An analysis of the pros and cons of using frozen versus fresh stem cell products in allogeneic stem cell transplantation. Bone Marrow Transplant. 2006;38:399-405.

6. Parody R, Caballero D, Marquez-Malaver FJ, Vazquez L, Saldana R, Madrigal $M D$, et al. To freeze or not to freeze peripheral blood stem cells prior to allogeneic transplantation from matched related donors. Eur J Haematol. 2013;91:448-55.

7. Ikebe C, Suzuki K. Mesenchymal stem cells for regenerative therapy: optimization of cell preparation protocols. Biomed Res Int. 2014;2014:951512.

8. Garvican ER, Cree S, Bull L, Smith RK, Dudhia J. Viability of equine mesenchymal stem cells during transport and implantation. Stem Cell Res Ther. 2014:5:94.

9. Ginis I, Grinblat B, Shirvan MH. Evaluation of bone marrow-derived mesenchymal stem cells after cryopreservation and hypothermic storage in clinically safe medium. Tissue Eng Part C Methods. 2012;18:453-63.

10. de Lima Prata K, de Santis GC, Orellana MD, Palma PVB, Brassesco MS, Covas DT. Cryopreservation of umbilical cord mesenchymal cells in xenofree conditions. Cytotherapy. 2012;14:694-700.

11. Adams MK, Goodrich LR, Rao S, Olea-Popelka F, Phillips N, Kisiday JD, et al Equine bone marrow-derived mesenchymal stromal cells (BMDMSCs) from the ilium and sternum: are there differences? Equine Vet J. 2013;45:372-5.

12. Koch TG, Heerkens T, Thomsen PD, Betts DH. Isolation of mesenchymal stem cells from equine umbilical cord blood. BMC Biotechnol. 2007;7:26.

13. Radcliffe $\mathrm{CH}$, Flaminio MJ, Fortier LA. Temporal analysis of equine bone marrow aspirate during establishment of putative mesenchymal progenitor cell populations. Stem Cells Dev. 2010;19:269-82.

14. Schnabel LV, Pezzanite LM, Antczak DF, Felippe MJ, Fortier LA. Equine bone marrow-derived mesenchymal stromal cells are heterogeneous in MHC class $\|$ expression and capable of inciting an immune response in vitro. Stem Cell Res Ther. 2014;5:13.

15. English A, Jones EA, Corscadden D, Henshaw K, Chapman T, Emery P, et al. A comparative assessment of cartilage and joint fat pad as a potential source of cells for autologous therapy development in knee osteoarthritis. Rheumatology (Oxford). 2007;46:1676-83.

16. Grogan SP, Barbero A, Winkelmann V, Rieser F, Fitzsimmons JS, O'Driscoll S, et al. Visual histological grading system for the evaluation of in vitro-generated neocartilage. Tissue Eng. 2006;12:2141-9.

17. Zhu Y, Ouyang Y, Chang Y, Luo C, Xu J, Zhang C, et al. Evaluation of the proliferation and differentiation behaviors of mesenchymal stem cells with partially converted borate glass containing different amounts of strontium in vitro. Mol Med Rep. 2013;7:1129-36.

18. Ivanov DP, Parker TL, Walker DA, Alexander C, Ashford MB, Gellert PR, et al. In vitro co-culture model of medulloblastoma and human neural stem cells for drug delivery assessment. J Biotechnol. 2015;205:3-13.

19. Begum J, Day W, Henderson C, Purewal S, Cerveira J, Summers H, Rees P, Davies D, Filby A. A method for evaluating the use of fluorescent dyes to track proliferation in cell lines by dye dilution. Cytometry A. 2013;12:1085-95

20. Zolnierowicz J, Ambrozek-Latecka M, Kawiak J, Wasilewska D, Hoser G. Monitoring cell proliferation in vitro with different cellular fluorescent dyes. Folia Histochem Cytobiol. 2013;51:193-200.

21. Portalska KJ, Groen N, Krenning G, Georgi N, Mentink A, Harmsen MC, et al. The effect of donor variation and senescence on endothelial differentiation of human mesenchymal stromal cells. Tissue Eng Part A. 2013;19:2318-29.

22. Haack-Sorensen M, Kastrup J. Cryopreservation and revival of mesenchymal stromal cells. Methods Mol Biol. 2011;698:161-74.

23. Jung J, Moon N, Ahn JY, Oh EJ, Kim M, Cho CS, et al. Mesenchymal stromal cells expanded in human allogenic cord blood serum display higher self-renewal and enhanced osteogenic potential. Stem Cells Dev. 2009;18:559-71.

24. Asghar W, El Assal R, Shafiee H, Anchan RM, Demirci U. Preserving human cells for regenerative, reproductive, and transfusion medicine. Biotechnol J. 2014;9:895-903 
25. Lovelock JE, Bishop MW. Prevention of freezing damage to living cells by dimethyl sulphoxide. Nature. 1959;183:1394-5.

26. Balint B, Ivanovic Z, Petakov M, Taseski J, Jovcic G, Stojanovic N, et al. The cryopreservation protocol optimal for progenitor recovery is not optimal for preservation of marrow repopulating ability. Bone Marrow Transplant. 1999;23:613-9.

27. Berz D, McCormack EM, Winer ES, Colvin GA, Quesenberny PJ. Cryopreservation of hematopoietic stem cells. Am J Hematol. 2007;82:463-72.

28. Weiner RS, Tobias JS, Yankee RA. The processing of human bone marrow for cryopreservation and reinfusion. Biomedicine. 1976;24:226-31.

29. Dariolli R, Bassaneze V, Nakamuta JS, Omae SV, Campos LC, Krieger JE. Porcine adipose tissue-derived mesenchymal stem cells retain their proliferative characteristics, senescence, karyotype and plasticity after long-term cryopreservation. PLoS One. 2013;8:e67939.

30. Liu Y, Xu X, Ma X, Martin-Rendon E, Watt S, Cui Z. Cryopreservation of human bone marrow-derived mesenchymal stem cells with reduced dimethylsulfoxide and well-defined freezing solutions. Biotechnol Prog. 2010;26:1635-43

31. Liu Y, Xu X, Ma XH, Liu J, Cui ZF. Effect of various freezing solutions on cryopreservation of mesenchymal stem cells from different animal species. Cryo Lett. 2011;32:425-35.

32. Luetzkendorf J, Nerger $\mathrm{K}$, Hering J, Moegel A, Hoffmann $\mathrm{K}$, Hoefers $\mathrm{C}$, et al. Cryopreservation does not alter main characteristics of Good Manufacturing Process-grade human multipotent mesenchymal stromal cells including immunomodulating potential and lack of malignant transformation. Cytotherapy. 2015;17:186-98

33. Tateishi K, Ando W, Higuchi C, Hart DA, Hashimoto J, Nakata K, et al. Comparison of human serum with fetal bovine serum for expansion and differentiation of human synovial MSC: potential feasibility for clinical applications. Cell Transplant. 2008;17:549-57.

\section{Submit your next manuscript to BioMed Central and take full advantage of:}

- Convenient online submission

- Thorough peer review

- No space constraints or color figure charges

- Immediate publication on acceptance

- Inclusion in PubMed, CAS, Scopus and Google Scholar

- Research which is freely available for redistribution 